\title{
Origin of High Velocity Pulsar and Soft Gamma Ray Repeaters
}

\author{
Hitoshi HANAMI \\ Physics Section, College of Humanities and Social Sciences, Iwate \\ University, Morioka 020, Japan
}

\begin{abstract}
We consider a close binary system with separation $\simeq 0.2 R_{\odot}$ which consists of a $(\mathrm{C}+\mathrm{O})$ star of mass $\simeq 4 \mathrm{M}_{\odot}$ and a neutron star as a progenitor of soft gamma-ray repeater (SGR) and a high velocity pulsar. After the event of the supernova explosion, both new and old neutron stars have high relative velocity of $\simeq 1000 \mathrm{~km} \mathrm{~s}^{-1}$ to the center of mass of the ejecta. SGR activities can be induced by the episodic accretion of the supernova ejecta onto the old neutron star or the strange high rotating pulsar of the new neutron star. Future observations with fine positional resolution can clarify the position ambiguity between the gamma ray and $\mathrm{X}$-ray sources.
\end{abstract}

\section{Introduction}

There are three soft gamma-ray repeaters SGR 0526-66, 1806-20 and 1900+14. As for SGR1806-20, several important observational results came out recently ( Kulkarni \& Frail 1993; Kouveliotou et al. 1994; Murakami et al. 1994; Kulkarni et al. 1994 ) The properties of SGR1806-20 remind us the similar coincidence of SGR0526-66 with the SNR N49 in Large Magellanic Cloud. All the above observational results suggest us the following common characteristics of SGR:

1 ) SGR is associated with a young ( $\leq 10,000$ year) plerion type supernova remnant and a rapidly spinning pulsar with spin period $\simeq 15 \mathrm{~ms}$ at birth.

2) The transverse velocity of the pulsar relative to the center of SNR is as high as $\simeq 1000 \mathrm{~km} \mathrm{~s}^{-1}$.

3) The location of SGR is coincident with the pulsar but they can be different objects with separation $5.5 \mathrm{pc}$ to $9 \mathrm{pc}$ corresponding to the inaccuracy in the determination of the position in gamma-ray (2 arcmin for SGR1806-20 and $20^{\prime \prime}$ for SGR0526-66, respectively).

One may incline to consider a model to explain these characteristics. SGR is associated with a rapidly spinning pulsar by which the plerion is powered, but Crab nebula which has a rapidly spinning $(\simeq 33 \mathrm{~ms})$ pulsar and is a plerion, for example, does not show activity of gamma-ray bursts at all. It is concluded that the maximum attainable velocities are around $200 \mathrm{~km} / \mathrm{s}$ by anisotropic supernova. So the transverse velocity of $\simeq 1000 \mathrm{~km} / \mathrm{s}$ is unusual. As this peculiarity, we consider that a progenitor of SGR is unusual, that is, we consider a close binary system with separation $\simeq 0.2 \mathrm{R}_{\odot}$ which consists of a $(\mathrm{C}+\mathrm{O})$ star of mass 
$\simeq 4 \mathrm{M}_{\odot}$ and a neutron star. We will argue how this model has a potential ability of explaining most observational results of SGR.

\section{High Transverse Velocity and SGR Activities}

Now we consider the transverse velocity given in the supernova event. Consider a system of an old neutron star 1 of mass $M_{1}$ at $\mathbf{r}_{1}$ and a $(\mathrm{C}+\mathrm{O})$ star 2 of mass $M_{2}$ at $\mathbf{r}_{2}$ with the separation $\left|\mathbf{r}_{1}-\mathbf{r}_{2}\right|_{0}=a$,.where the subscript 0 means the value at the $\mathrm{SN}$ explosion. When the $\mathrm{SN}$ explosion of the $(\mathrm{C}+\mathrm{O})$ star occurs in this binary, the stellar system obtains high velocity. The relative velocity between the center of mass of the neutron stars and the SNR becomes

$$
V_{N S-S N R}=\frac{M_{1}}{M_{t}^{\prime}} \Omega_{o r b} a=1.16 \times 10^{8} \mathrm{cms}^{-1}\left(\frac{M_{1}+M_{2}}{5.6 M_{\odot}}\right)^{0.5}\left(\frac{a}{1.4 \times 10^{10} \mathrm{~cm}}\right)^{-0.5} .
$$

Thus in our model the relative velocity of new pulsar and the SNR can be as high as $\simeq 1000 \mathrm{kms}^{-1}$.

In our scenario, we consider the episodic accretion onto the old neutron star 1 as the origin of SGR (Hanami et al. 1995). The typical energy of burst is determined by the mass of the accreting planetesimal. Recent events for SGR1806-20 needs the accretion of the mass $\simeq 10^{19} \eta^{-1} g$ onto the neutron star 1 for the burst energy, where $\eta$ is the energy conversion efficiency from the gravitational energy to the observed gamma-ray radiation.

\section{Conclusions}

Since our scenario implies that SGR progenitor may be a $(C+O)$ star - neutron star binary which is also the possible candidate for a double neutron star system (e.g. PSR 1913+16, 1534+12) (Yamaoka et al. 1993). This type of system should explode twice as the evolution from HMXB. ¿From the observed number and the life time $\leq 10^{4} \mathrm{yr}$ for SGRs, the birth rate is $\geq 10^{-4} \mathrm{yr}^{-1}$ in our galaxy. This rate is comparable to the birth rate $\simeq 5 \times 10^{-4} \mathrm{yr}^{-1}$ of HMXB (Pols et al. 1991). So our scenario suggests that $\simeq 20 \%$ of $\mathrm{HMXB}$ evolves to SGR. This formation rate, in our model, means that a galaxy may eject $10^{6}$ old neutron stars with $10^{-5} M_{\odot}$ dust disks to $10 M p c$ in $10^{10} \mathrm{yr}$. The object unbound to galaxies might have something to do with origins of classical gamma ray bursts.

\section{References}

Frail, D.A., Kulkarni, S.R. \& Vasisht, G. 1993, Nature, 365, 136

Hanami, H. et al. 1992, Prog. Theo. Phys., 94, 1001

Kouveliotou, C. et al. 1994, Nature, 368, 125

Kulkarni, S.R. \& Frail, D.A. 1993, Nature, 365, 33

Kulkarni, S.R. et al. 1994, Nature, 368, 129

Yamaoka H., Shigeyama, T. and Nomoto, K. 1993, A\&A, 267, 433 